with acute ST-segment elevation myocardial infarction (STEMI) and 44 patients with stable coronary artery disease (CAD). Overall, 54 differentially expressed transcripts were isolated from STEMI and CAD patients. CD69 and MRP-14 were the most significantly increased probe sets in STEMI, with CD69 showing 2.7-fold higher, and MRP-14 showing 2.2-fold higher, median expression than in CAD patients.

A prospective, case-control study was used to validate the MRP-14 candidate gene product, to assess the risk of future cardiovascular events according to baseline plasma levels of the MRP-8/14 heterodimer in apparently healthy women (255 case-control pairs). Women who developed cardiovascular events over a median follow-up of 2.9 years had significantly higher baseline MRP-8/14 levels than women without such events $(P<0.001)$. Women with MRP-8/14 levels in the highest quartile had a 3.8-fold increased risk of any vascular event compared with those in the lowest quartile $(P<0.001)$; increased risk was independent of standard risk factors and C-reactive protein level.

Increasing plasma concentrations of MRP8/14 among healthy individuals might, therefore, be useful for predicting future cardiovascular events. Additional analyses of differentially expressed platelet transcripts are needed to further our understanding of platelet function in health and disease.

Original article Healy AM et al. (2006) Platelet expression profiling and clinical validation of myeloid-related protein-14 as a novel determinant of cardiovascular events. Circulation 113: $2278-2284$

\section{Evidence for statin-mediated functions independent of lipid lowering}

Statins have been shown to have beneficial effects on endothelial function, in addition to having anti-inflammatory and immunomodulatory properties. Whether such pleiotropic functions of statins are independent of their effects on cholesterol reduction, however, is unclear. Fichtlscherer et al. have begun to unravel the mechanisms of statinmediated functions by comparing the effects of short-term cholesterol reductions with either atorvastatin or ezetimibe-a novel cholesterol absorption inhibitor-on endothelial function in patients with coronary artery disease.
A total of 60 patients with coronary artery disease were recruited to the study. Patients who had not been previously treated with statins were randomized to receive either $10 \mathrm{mg} /$ day ezetimibe or $40 \mathrm{mg} /$ day atorvastatin. Individuals who had received $20 \mathrm{mg}$ of simvastatin per day for at least 6 months received an additional $10 \mathrm{mg} /$ day ezetimibe. Finally, those patients who had previously received chronic atorvastatin therapy at $10 \mathrm{mg} /$ day had their daily dose increased to $40 \mathrm{mg}$. Venous occlusion plethysmography was used to measure patients' forearm blood flow response before and after therapy.

Despite comparable and significant reductions in LDL cholesterol levels after 4 weeks, neither ezetimibe monotherapy nor ezetimibe combined with simvastatin were associated with an increase in acetylcholine-mediated forearm blood flow response. Only those patients who had received atorvastatin therapy demonstrated improved endothelial vasodilator function in the forearm circulation. These findings indicate that mechanisms beyond LDL cholesterol reduction have a role in the beneficial effects of statin therapy on endothelial vasodilator function.

Original article Fichtlscherer S et al. (2006) Differential effects of short-term lipid lowering with ezetimibe and statins on endothelial function in patients with CAD: clinical evidence for 'pleiotropic' functions of statin therapy. Eur Heart J 27: 1182-1190

\section{Community study shows shift in distribution of mortality from cardiovascular disease}

While there has been a downward trend in ageadjusted cardiovascular disease (CVD) mortality over the last few decades, there is evidence to suggest age and sex disparity. Further controversy surrounds the magnitude of the decline for in-hospital versus out-of-hospital deaths and CVD categories other than coronary heart disease (CHD).

To address these issues, Gerber et al. studied CVD mortality trends in Olmsted County, MN over a 25-year period from 1979 to 2003. Date of birth, date of death, sex, underlying cause of death, and site of death (i.e. in-hospital or out-ofhospital) were collected from death certificates. The American Heart Association classification was used to divide CVD mortality into three 